\title{
A phase II study of sequential chemotherapy with docetaxel after the weekly PELF regimen in advanced gastric cancer. A report from the Italian group for the study of digestive tract cancer
}

\author{
S Cascinu ${ }^{1}$, F Graziano ${ }^{2}$, S Barni ${ }^{3}$, R Labianca ${ }^{4}$, G Comella ${ }^{5}$, R Casaretti ${ }^{5}$, L Frontini ${ }^{6}$, V Catalano ${ }^{7}$, AM Baldelli and $^{2}$ \\ G Catalano ${ }^{7}$
}

1Division of Medical Oncology, Azienda Ospedale di Parma, Italy; ${ }^{2}$ Medical Oncology Unit, Azienda Ospedale di Urbino, Italy; ${ }^{3}$ Division of Medical Oncology, Azienda Ospedale di Treviglio, Italy; ${ }^{4}$ Medical Oncology Unit, Azienda Ospedale di Bergamo, Italy; ${ }^{5}$ National Tumor Institute, Naples, Italy; ${ }^{6}$ Medical Oncology Unit, Ospedale San Paolo, Milan, Italy; ${ }^{7}$ Division of Medical Oncology, Azienda Ospedale di Pesaro, Italy

\begin{abstract}
Summary In advanced gastric cancer, we investigated feasibility and activity of sequential chemotherapy with docetaxel after an intensive weekly regimen consisting of cisplatin, epidoxorubicin, fluorouracil, leucovorin (PELF) plus filgrastim. Chemotherapy-naive patients with relapsed or metastatic gastric cancer received 8 weekly administrations of chemotherapy with cisplatin $40 \mathrm{mg} / \mathrm{m}^{2}$, fluorouracil $500 \mathrm{mg} / \mathrm{m}^{2}, \mathrm{epi}$ doxorubicin $35 \mathrm{mg} / \mathrm{m}^{2}$, 6S-steroisomer of leucovorin $250 \mathrm{mg} / \mathrm{m}^{2}$ and glutathione $1.5 \mathrm{~g} / \mathrm{m}^{2}$. On the other days filgrastim $5 \mu \mathrm{g} \mathrm{kg}^{-1}$ was administered by subcutanous injection. Subsequently, patients with partial response or stable disease received 3 cycles of docetaxel 100 $\mathrm{mg} / \mathrm{m}^{2}$ every 3 weeks. 40 patients have been enrolled and they are evaluable for response and toxicity. After the PELF regimen, 3 patients achieved complete response, 13 patients showed partial response, 21 patients had stable disease and 3 patients progressed (40\% response rate; $95 \% \mathrm{Cl} 25 \%$ to $55 \%$ ). After docetaxel, 9 out 34 patients improved the outcome (26.5\%); 7 patients with stable disease achieved partial response and 2 patients with partial response achieved complete response. The overall response rate in the 40 patients was $57.5 \%(95 \% \mathrm{Cl}$, $42.5 \%$ to $72.5 \%$ ). The PELF regimen did not cause any grade IV toxicity, the most frequent grade III acute side-effects were thrombocytopenia and vomiting which occurred in the $10 \%$ of 320 PELF cycles. Docetaxel caused grade III-IV neutropenia and thrombocytopenia in the $10 \%$ and the $19 \%$ of cycles respectively. Fatigue was a frequent side-effect during both PELF and docetaxel chemotherapy. The sequential application of docetaxel after PELF chemotherapy gained major objective responses with manageable toxicity. This strategy is worth of further investigation in the setting of palliative or neoadjuvant chemotherapy. (C) 2001 Cancer Research Campaign http://www.bjcancer.com
\end{abstract}

Keywords: gastric cancer; sequential chemotherapy; docetaxel

Gastric cancer is considered a chemosensitive disease and second generation combination chemotherapy regimens have produced high response rates and impressive survival times (Hill and Cunningham, 1998). Unfortunately, about half of the patients treated with chemotherapy is unresponsive, and less than half of the patients with locally advanced disease is amenable of surgical resection after neoadjuvant chemotherapy. For these reasons, new and hopefully more effective drugs, or innovative treatment strategies are needed.

Docetaxel is a semisynthetic taxoid with cytotoxic activity against a broad spectrum of human solid tumors (Cortes and Pazdur, 1995). Docetaxel has been tested in advanced gastric cancer and it showed promising single-agent activity with $20 \%$ to $24 \%$ response rates in treated and chemotherapy-naive patients (Sulkes et al, 1994; Furue and Taguchi, 1998; Mavroudis et al, 1999; Vanhofer et al, 1999). A logical step of investigation consisted in the development of multi-drug schedules including docetaxel and other known active drugs. In early phase II studies,

Received 27 July 2000

Revised 1 November 2000

Accepted 21 November 2000

Correspondence to: S Cascinu combinations of docetaxel with cisplatin, fluorouracil or epidoxorubicin showed promising results, but neutropenia and nonhaematologic toxicity were often significant (Ajani et al, 1999; Andrè et al, 1999; Roth et al, 2000).

Ongoing studies are exploring new schedules of docetaxelbased combination chemotherapy to ameliorate the efficacy/toxicity ratio. Sequential schedules may maximize the dose-intensity of each single agent and avoid the overlapping toxicity caused by the concomitant administration of active drugs. Safety and efficacy of sequential chemotherapy with docetaxel has been tested in breast cancer with favourable results (Antoine et al, 1998), and this chemotherapeutic strategy deserves investigation in other tumors with documented activity of docetaxel (Pronk et al, 1995).

In advanced gastric cancer, we investigated safety and activity of sequential chemotherapy with docetaxel after the intensive weekly PELF regimen (Cascinu et al, 1997, 1998).

\section{MATERIALS AND METHODS}

\section{Patient selection}

Chemotherapy-naive patients with pathologically confirmed, relapsed or metastatic gastric cancer were considered eligible for 
the study. Other eligibility criteria were: Eastern Cooperative Oncology Group (ECOG) performance status 0 , 1, or 2; age equal or less than 75 years; normal liver, renal, and bone marrow functions. The protocol was approved by each local institutional review board and all patients gave written informed consent.

\section{Treatment plan}

PELF chemotherapy consisted of a 1 day per week administration of cisplatin $40 \mathrm{mg} / \mathrm{m}^{2}$, fluorouracil $500 \mathrm{mg} / \mathrm{m}^{2}$, epi-doxorubicin $35 \mathrm{mg} / \mathrm{m}^{2}$, 6S-stereoisomer of leucovorin $250 \mathrm{mg} / \mathrm{m}^{2}$ and glutathione $1.5 \mathrm{~g} / \mathrm{m}^{2}$. All drugs were given intravenously and on the other days filgrastim was administered by subcutanous injection at a dose of $5 \mu \mathrm{g} \mathrm{kg}^{-1}$ (Cascinu et al, 1997). After 8 weekly cycles patients were re-evaluated and those with partial response or stable disease received docetaxel $100 \mathrm{mg} / \mathrm{m}^{2}$ via a 1-hour intravenous infusion every 3 weeks. After 3 cycles with docetaxel patients were re-evaluated for response to the sequential treatment. All patients received emesis prophylaxis with 5-HT3 inhibitors and hyperhydration during each course of PELF chemotherapy. Patients who received docetaxel were treated with dexamethasone $8 \mathrm{mg}$ p.o. administered 12 and 6 hours before drug infusion and $8 \mathrm{mg}$ twice daily for an additional 4 days.

Full doses of the anticancer drugs were given if the neutrophil count was equal or $>1.5 \times 10^{9} 1^{-1}$ and the platelet count equal or $>100 \times 10^{9} 1^{-1}$; dose reductions were not recommended and values less than these necessitated a 7-day treatment delay. Patients treated with docetaxel did not receive prophylactic haematopoietic growth factors. However, filgrastim was employed in patients with grade III neutropenia lasting more than one week or grade IV neutropenia, so that treatment at the $100 \mathrm{mg} / \mathrm{m}^{2}$ dose level could be maintained.

\section{Evaluation procedures}

Pretreatment evaluation consisted of baseline studies including: medical history, physical examination, blood chemistries, urinoanalysis and ECG. Also, chest X-rays, abdominal computed tomography or magnetic resonance, bone scan and any other test to identify the extent of disease was performed. These studies were repeated after 8 weekly administration of PELF chemotherapy, after 3 cycles of docetaxel and every 3 months thereafter. Responses to the sequential program were not confirmed by an early repeat estimation.

All patients had physical examination and biochemical profile before each administration of chemotherapy. Response and toxicity were evaluated and graduated according to the standard World Health Organization (WHO) criteria (Miller et al, 1981). Patients treated with docetaxel with no fluid retention were considered grade 0 ; asymptomatic weight gain, grade 1; mild peripheral oedema that did not require diuretics, grade 2; symptomatic, moderate edema tha required diuretics, grade 3; edema/fluid retention that necessitated docetaxel withdrawal, grade 4 .

\section{Statistical plan}

The optimal two-stage design was adopted for this phase II trial (Simon, 1989). The minimum target activity level was a $20 \%$ gain in objective responses attained by docetaxel after the PELF regimen. Early discontinuation of the study was planned in the case of no response in the first 12 assessable patients treated with docetaxel ( $\alpha$ and $\beta$ error probabilities 0.010 and 0.010 ). Alternatively, a planned sample size of approximately 30 patients was chosen to better estimate efficacy; $35 \%$ maximum width of the $95 \%$ confidence interval (CI) for the overall response rate. Time to disease progression was measured from date of registration to the date of progressive disease. Overall survival was measured form the time of registration to the date of death resulting from any cause.

\section{RESULTS}

Between October 1998 and November 199940 patients entered this study and they are fully evaluable for response and toxicity. Their characteristics are reported in Table 1.

The toxicity profile of the PELF regimen was acceptable and it was similar to that of previous studies. None of the 40 patients suffered from grade IV toxicity and 12 patients (35.3\%) experienced acute grade III adverse events. The major grade III toxicities were thrombocytopenia and vomiting which occurred in the $10 \%$ of 320 PELF cycles (Table 2). The most frequent chronic adverse events attributable to the PELF regimen (Table 3) were grade III alopecia in the $88 \%$ of the patients and grade II asthenia in the $25 \%$ of the patients. Transient grade II peripheral neuropathy occurred in 2 patients. All the 40 patients received eight cycles, but due to neutropenia and/or thrombocytopenia, the $30 \%$ of 320 PELF administrations were delayed a week. After the PELF regimen, 3 patients achieved complete response, 13 patients showed partial response, 21 patients had stable disease and 3 patients progressed (40\% response rate; $95 \%$ CI $25 \%$ to $55 \%$ ).

According to the treatment protocol 34 patients started docetaxel and all of them completed 3 cycles of chemotherapy. Acute adverse events in 102 cycles are listed in Table 4; grade III-IV neutropenia and thrombocytopenia occurred in the $10 \%$ and the $19 \%$ of cycles, respectively. None of the patients experienced neutropenic fever or sepsis, but 10 patients with grade III/IV neutropenia were treated with prophylactic filgrastim to maintain the planned dose of chemotherapy. Due to neutropenia and/or thrombocytopenia, docetaxel was delayed a week in the $45 \%$ of 102 cycles. One patient with grade III dermatitis had 50\% dose reduction in the last administration of docetaxel. Asthenia was the most frequent chronic adverse events (Table 5) and it resulted grade I/II in 18 patients and grade III in 2 patients. None of the

Table 1 Characteristics of the 40 patients enrolled in the study

\begin{tabular}{lc}
\hline Number of patients & 40 \\
Sex ratio M/F & $23 / 17$ \\
Median age (range) & 57 y (38-69) \\
ECOG performance status & \\
0 & 8 \\
I & 22 \\
II & 10 \\
Prior surgery & \\
None & 5 \\
Curative & 22 \\
Palliative & 13 \\
Disease sites: & \\
Liver & 14 \\
Lymph nodes + abdominal mass & 13 \\
Liver + lymph nodes & 5 \\
Local relapse & 5 \\
Lung + liver & 2 \\
Lung & 1 \\
\hline
\end{tabular}


Table 2 Acute adverse events associated with the PELF regimen in 320 cycles

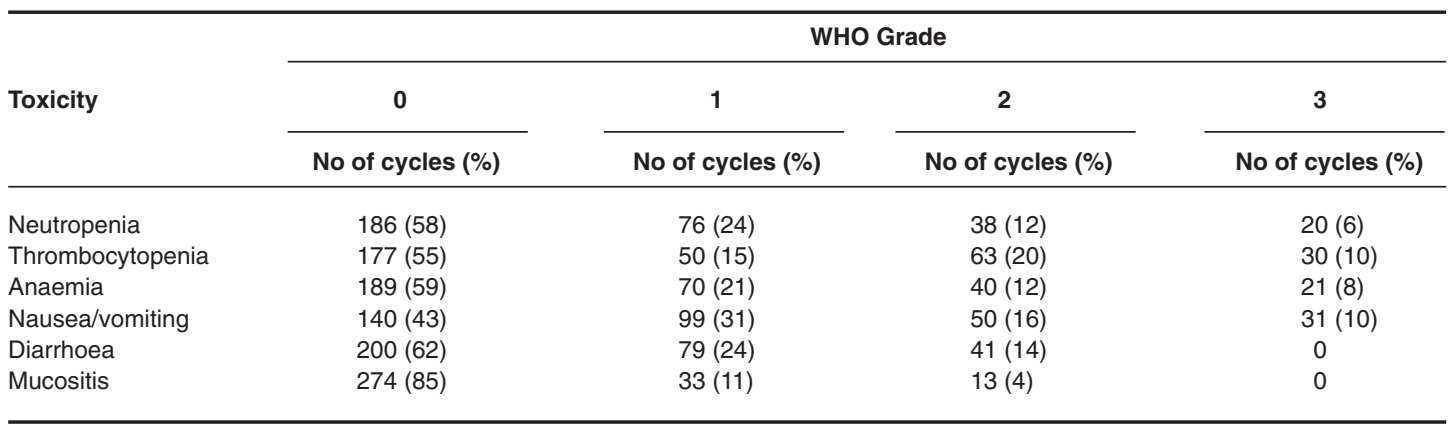

None of the patients treated with the PELF regimen experienced grade 4 side-effects.

Table 3 Chronic adverse events associated with the PELF regimen in 40 enrolled patients

\begin{tabular}{|c|c|c|c|c|}
\hline \multirow{3}{*}{ Toxicity } & \multicolumn{4}{|c|}{ WHO Grade } \\
\hline & 0 & 1 & 2 & 3 \\
\hline & No of pts (\%) & No of pts (\%) & No of pts (\%) & No of pts (\%) \\
\hline Asthenia & $22(55)$ & $8(20)$ & $10(25)$ & 0 \\
\hline Peripheral neurotoxicity & $32(80)$ & $6(15)$ & $2(5)$ & 0 \\
\hline Nail toxicity & $33(82)$ & $4(10)$ & $3(8)$ & 0 \\
\hline Constipation & $20(50)$ & $20(50)$ & 0 & 0 \\
\hline Alopecia & 0 & 0 & $5(12)$ & $35(88)$ \\
\hline
\end{tabular}

None of the patients treated with the PELF regimen experienced grade 4 side-effects.

Table 4 Acute adverse events attributable to docetaxel in 102 cycles

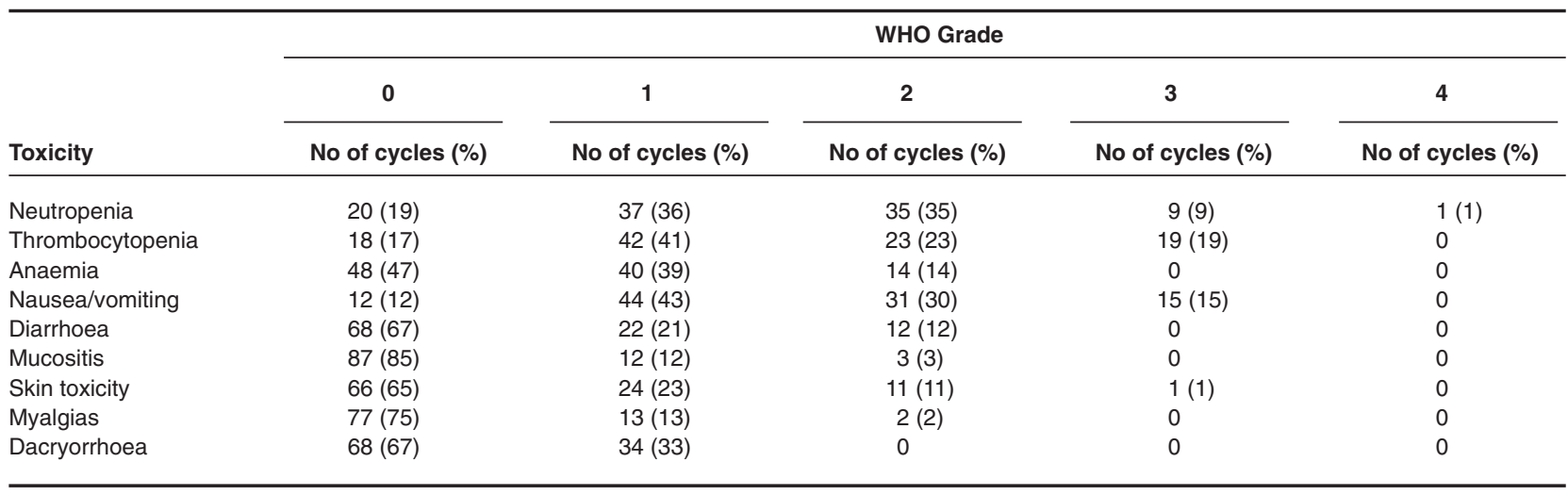

Table 5 Chronic adverse events attributable to docetaxel in 34 patients

\begin{tabular}{|c|c|c|c|c|}
\hline \multirow[b]{3}{*}{ Toxicity } & \multicolumn{4}{|c|}{ WHO Grade } \\
\hline & 0 & 1 & 2 & 3 \\
\hline & No of pts (\%) & No of pts (\%) & No of pts (\%) & No of pts (\%) \\
\hline Asthenia & $14(42)$ & $8(23)$ & $10(29)$ & $2(6)$ \\
\hline Peripheral neurotoxicity & $28(82)$ & $2(6)$ & $4(12)$ & 0 \\
\hline Fluid retention & $29(85)$ & 0 & $5(15)$ & 0 \\
\hline Constipation & $30(88)$ & $4(12)$ & 0 & 0 \\
\hline Nail toxicity & $68(67)$ & $22(21)$ & $4(12)$ & 0 \\
\hline Alopecia & 0 & 0 & $2(6)$ & $32(94)$ \\
\hline
\end{tabular}

Chronic grade 4 side-effects were not observed in patients receiving docetaxel. 
patients experienced hypersensitivity reactions but moderate fluid retention syndrome was observed in 5 patients.

None of the 34 patients progressed during docetaxel chemotherapy and 9 of them (26.5\%) gained a major response; 7 patients with stable disease improved to partial response and 2 patients with partial response achieved complete response. Excluding from the overall response rate the two patients who improved partial response to complete response, the PELFdocetaxel regimen produced unconfirmed objective responses in 23 out of 40 patients $(57.5 \%$ response rate with $95 \%$ CI, $42.5 \%$ to $72.5 \%$ ). Time to disease progression and median survival time resulted 7 months and 12.6 months, respectively.

\section{DIsCusSION}

Recent phase II studies have established the role of docetaxel in first-line and second-line treatment of advanced gastric cancer (Sulkes et al, 1994; Furue and Taguchi, 1998; Mavroudis et al, 1999). As a consequence, the hope for more potent regimens prompted several investigators to evaluate docetaxel in multidrug regimens. Early investigations of polychemotherapy with docetaxel, cisplatin and fluorouracil yelded high response rates, but side-effects were often pronounced due to overlapping toxicity (Andrè et al, 1999; Ajani et al, 2000; Roth et al, 2000). Roth et al (2000) reported a $56 \%$ response rate with the docetaxel-cisplatin combination. However, this schedule caused relevant haematologic toxicity with a high number of grade III/IV episodes of neutropenia ( $80 \%$ of the cycles) and non-fatal febrile neutropenia in $19 \%$ of the patients. Ajani et al (2000) treated patients with advanced gastric cancer with the combination of docetaxel, cisplatin and fluorouracil. The incidence of grade III/IV neutropenia was $72 \%$ per cycle. In addition, grade III/IV stomatitis was observed in the $16 \%$ of cycles. Available data suggest that about $20 \%$ to $30 \%$ of patients treated with these regimens do not receive the treatment on schedule for dose reductions or delays, and up to the $20 \%$ of patients discontinue therapy due to toxicity.

Combining old anti-cancer drugs with new compounds is a formidable challenge which requires several attempts to optimize the efficacy/toxicity ratio. Sequential chemotherapy (Day, 1986) and dose-dense schedules (Fizazi and Zelek, 2000) may offer this opportunity. In the present experience, patients received first-line chemotherapy with four of the most active drug in advanced gastric cancer, and the toxicity profile of the PELF-docetaxel chemotherapy seemed more favourable than that of new combinations using a concomitant administration of drugs. Interestingly, sequential docetaxel caused more episodes of grade II neutropenia (35\% vs $12 \%$ of cycles) and grade III thrombocytopenia (19\% vs $10 \%$ of cycles) than the PELF induction. Also, fatigue was more frequent after chemotherapy with docetaxel. According to our protocol, filgrastim was used after every cycle of the PELF regimen, whilst it was employed in the case of grade III-IV neutropenia during docetaxel chemotherapy. This may explain differences in the number of cycles with neutropenia between the PELF regimen and docetaxel chemotherapy. Also, it is possible that patients receiving the sequential programme were more likely to experience side-effects due to the prolonged exposure to chemotherapy with cumulative toxicity.

In the first phase II study (Cascinu et al, 1997), the PELF regimen showed $62 \%$ overall response rate which dropped to $46 \%$ in a subsequent analysis in patients with locally advanced disease (Cascinu et al 1998). In the present study, PELF chemotherapy alone achieved $40 \%$ response rate which approached $60 \%$ after docetaxel. The well-known ECF regimen showed 71\% response rate in early phase II studies (Findlay et al, 1994) which dropped to $45 \%$ in a randomized trial (Webb et al, 1997). Phase III trials allows a proper analysis of response rates, survival, adverse events and quality of life and they are necessary for the testing of secondgeneration chemotherapy regimens in advanced gastric cancer. The non-randomized design of the PELF trials does not allow any definitive conclusion and any direct comparison for efficacy. The high response rate showed by the PELF chemotherapy in the early investigation needs to the confirmed in a randomized study, and a comparison with the PELF-docetaxel sequence would be of interest. At present, we may consider the PELF-docetaxel as an interesting evolution of the PELF regimen; this new sequential combination showed a favourable toxicity/efficacy ratio and it deserves further investigation in the palliative or neoadjuvant setting.

New combination chemotherapy regimens with substantial response rates and moderate toxicity may be studied as neoadjuvant chemotherapy (Kelsen, 1996). After PELF chemotherapy, 13 out of 32 patients with unresectable, locally advanced disease underwent surgery and their tumour was completely removed (Cascinu et al, 1998). Toxicity was acceptable, neither treatmentrelated deaths, nor surgical complications were observed. Also the ECF regimen was employed in the neoadjuvant setting (Findlay et al, 1994; Melcher et al, 1996). In these experiences, the ECF chemotherapy showed mild toxicity, and it allowed surgery in about half of patients with locally advanced disease.

An innovative strategy of neoadjuvant chemotherapy should consider the identification of patients with chemosensitive disease (Reichle et al, 2000). In fact, sequential chemotherapy may display the population of patients who may ulteriorly respond to non-cross resistant agents. In our previous experience with the PELF regimen (Cascinu et al, 1997), all patients but one achieved a maximum response after 8 cycles and 6 more cycles of the same chemotherapy did not improve the outcome. In the present study, docetaxel following PELF induction gained major responses, moreover, patients who improved after docetaxel had a major tumor shrinkage rather than a simple turn of minor response to partial response. This effect may be beneficial in a population of patients with locally advanced disease, by increasing the chance of successful surgical resection.

In conclusion, the discovery of new active compounds and their testing in multi-drug regimens has allowed progresses in the medical management of gastric cancer. Future trials will confirm or not the superiority of second-generation polychemotherapy regimens and their role in the palliative or the neoadjuvant setting. In this perspective, the PELF-docetaxel chemotherapy is worth of further investigations and we are planning a phase II analysis in neoadjuvant chemotherapy and a randomized trial of PELF vs PELF-docetaxel in metastatic disease.

\section{REFERENCES}

Ajani JA, Fodor M, Van Cutsem E, Tjulandin S, Moiseyenko V, Cabral F, Majilis A, Chao Y, Zuber A, Blattmann C, Garay C and Jacques C (2000) Multinational randomized phase II trial of docetaxel and cisplatin with or without 5-fluorouracil in patients with advanced gastric cancer or GE junction adenocarcinoma. Proc Am Soc Clin Oncol 19: 247

Andrè T, Louvet C, Ychou M, Gamelin E, Mousseau E, Carola S, Assadourian S and De Gramont A (1999) Docetaxel-epirubicin as second-line treatment for patients with advanced gastric cancer. Proc Am Soc Clin Oncol 18: 277 
Antoine EC, Chollet P, Monfardini S, Sorio R, Ambrosini G, Benhammouda A, Mazen MF, Ramazeilles C, Azli N and Khayat D (1998) Sequential administration of docetaxel followed by AC in first-line metastatic breast cancer patients: final results. Ann Oncol 9 (4): 19

Cascinu S, Labianca R, Alessandroni P, Marcellini M, Silva RR, Pancera G, Testa E, Martignoni G, Barni S, Frontini L, Zaniboni A, Luporini G, Cellerino R and Catalano G (1997) Intensive weekly chemotherapy for advanced gastric cancer using fluorouracil, cisplatin, epidoxorubicin, 6S-leucovorin, gluthatione and filgrastim: a report of the Italian Group for the Study of the Digestive Tract Cancer. J Clin Oncol 15: 3313-3319

Cascinu S, Labianca R, Graziano F, Pancera G, Barni S, Frontini L, Luporini G, Cellerino R and Catalano G (1998) Intensive weekly chemotherapy for locally advanced gastric cancer using 5-fluorouracil, cisplatin, epidoxorubicin, 6S-leucovorin, gluthatione and filgrastim: a report from the Italian Group for the Study of Digestive Tract Cancer (GISCAD). Br J Cancer 78: 390-393

Cortes JE and Pazdur R (1995) Docetaxel. J Clin Oncol 13: 2643-2655

Day RS (1986) Treatment sequencing, asymmetry and uncertainty: protocol strategies for combination chemotherapy. Cancer Res 46: 3876-3885

Findlay M, Cunningham D, Norman A, Mansi J, Nicolson M, Hickish T, Nicolson V, Nash A, Sacks N and Ford H (1994) A phase II study in advanced gastro-esophageal cancer using epirubicin and cisplatin in combination with continuous infusion 5-fluorouracil (ECF). Ann Oncol 5: 609-616

Fizazi K and Zelek L (2000) Is one cycle every three or four weeks obsolete? A critical review of dose-dense chemotherapy in solid neoplasms. Ann Oncol 11: 133-149

Furue H and Taguchi T (1998) A late phase II study of RP56976 (docetaxel) in patients with advanced or recurrent gastric cancer. Ann Oncol 9 (supp.4): 49

Hill ME and Cunningham D (1998) Medical management of advanced gastric cancer. Cancer Treat Rev 24: 113-118

Kelsen DP (1996) Adjuvant and neoadjuvant therapy for gastric cancer. Semin Oncol 23: $379-389$

Mavroudis D, Kakolyris S, Kouroussis Ch, Androulakis N, Agelaki S, Kalbakis K, Sarra E, Vardakis N, Souglakos J, Hatzidaki D, Malliotakis P, Samonis G and Geogoulias G (1999) First line treatment of advanced gastric cancer with docetaxel monotherapy and granulocyte colony-stimulating factor (G-CSF). Proc Am Soc Clin Oncol 18: 254

Melcher AA, Mort D and Maughan TS (1996) Epirubicin, cisplatin and continuous infusion 5-fluorouracil (ECF) as neoadjuvant chemotherapy in gastro-oesophageal cancer. Br J Cancer 74: 1651-1654

Miller AB, Hoogstraten B, Staquet M and Winkler V. Reporting results of cancer treatment (1981) Cancer 47: 207-214

Pronk LC, Stoter G and Verweij J (1995) Docetaxel (Taxotere): single agent activity, development of combination treatment and reducing side-effects. Cancer Treat Rev 21: 463-478

Reichle A, Jauch K, Hofstaedter F, Bataille F, Erdmann A, Kreuser E and Andreesen R (2000) Preoperative chemotherapy and consecutive gastrectomy in chemosensitive gastric cancer. Testing a new strategy. Proc Am Soc Clin Oncol 19: 298

Roth AD, Maibach R, Martinelli G, Fazio N, Aapro MS, Pagani O, Morant R, Borner MM, Herrmann R, Honegger H, Cavalli F, Alberto P, Castiglione M and Goldhirsch A (2000) Docetaxel (Taxotere)-cisplatin (TC): an effective drug combination in gastric carcinoma. Ann Oncol 11: 301-306

Simon R (1989). Optimal two-stage designs for phase II clinical trials. Controlled Clin Trials 10: $1-10$

Sulkes A, Smyth J, Sessa C, Dirix LY, Vermorken JB, Kaye S, Wanders J, Franklin H, LeBail N and Verweij J (1994) Docetaxel (Taxotere) in advanced gastric cancer: results of a phase II clinical trial. $\mathrm{Br} J$ Cancer 70: $380-383$

Vanhoefer U, Wilke H, Harstrick A, Achterrath W, Preusser P, Sthal M, Clemens MR, Thiel E, Flasshove M, Fink U, Trenn G and Seeber S (1999) Phase II study of docetaxel as second line chemotherapy in metastatic gastric cancer. Proc Am Soc Clin Oncol 18: 303

Webb A, Cunningham D, Scarffe JH, Harper P, Norman A, Joffe JK, Hughes M, Mansi J, Findlay M, Hill A, Oates J, Nicolson M, Hickish T, O’Brien M, Iveson T, Watson M, Underhill C, Wardley A and Meehan M (1997) Randomized trial comparing epirubicin, cisplatin, and fluorouracil versus fluorouracil, doxorubicin, and methotrexate in advanced esophagogastric cancer. J Clin Oncol 15: 261-267 